\title{
LIR: Longwave Infrared Camera onboard the Venus orbiter Akatsuki
}

\author{
Tetsuya Fukuhara ${ }^{1}$, Makoto Taguchi ${ }^{2}$, Takeshi Imamura ${ }^{3}$, Masato Nakamura ${ }^{3}$, Munetaka Ueno ${ }^{3}$, Makoto Suzuki ${ }^{3}$, \\ Naomoto Iwagami ${ }^{4}$, Mitsuteru Sato ${ }^{1}$, Kazuaki Mitsuyama ${ }^{4}$, George L. Hashimoto ${ }^{5}$, \\ Ryo Ohshima ${ }^{4}$, Toru Kouyama ${ }^{4}$, Hiroki Ando ${ }^{4}$, and Masahiko Futaguchi ${ }^{2}$ \\ ${ }^{1}$ Department of Earth Sciences, Hokkaido University, North-10 West-8, Sapporo, Hokkaido 060-0810, Japan \\ ${ }^{2}$ Department of Physics, Rikkyo University, 3-34-1 Nishi-ikebukuro, Toshima-ku, Tokyo 171-8501, Japan \\ ${ }^{3}$ Institute of Space and Astronautical Science, Japan Aerospace Exploration Agency, \\ 3-1-1 Yoshinodai, Chuo-ku, Sagamihara, Kanagawa 252-5210, Japan \\ ${ }^{4}$ Department of Earth and Planetary Science, The University of Tokyo, 7-3-1 Hongo, Bunkyo-ku, Tokyo 113-0033, Japan \\ ${ }^{5}$ Department of Earth Sciences, Okayama University, 3-1-1 Tsushima-Naka, Kita-ku, Okayama 700-8530, Japan
}

(Received June 9, 2010; Revised March 20, 2011; Accepted June 9, 2011; Online published January 26, 2012)

The Longwave Infrared Camera (LIR) is one of a suite of cameras onboard the Venus orbiter Akatsuki. It will take images of thermal radiation in the wavelength range of $8-12 \mu \mathrm{m}$ emitted by the Venus cloud tops. The use of an uncooled micro-bolometer array as an infrared image sensor makes LIR a lightweight, small and lowpower consumption instrument with a required noise equivalent temperature difference of $0.3 \mathrm{~K}$. Temperature and horizontal wind fields at the cloud-top will be retrieved for both dayside and nightside with equal quality. This will provide key observations to understand the mechanism of super rotation and the thermal budget of the planet. LIR will also monitor variations of the polar dipole and collar which are characteristic thermal features in the Venusian atmosphere. Mechanisms of the upper-cloud formation will be investigated using sequences of close-up images. The morphology of the nightside upper cloud will be studied in detail for the first time.

Key words: Venus, Planet-C, atmosphere.

\section{Introduction}

The energy budget, dynamics and chemical cycle of the Venusian atmosphere is strongly influenced by the $\mathrm{H}_{2} \mathrm{SO}_{4}$ $\mathrm{H}_{2} \mathrm{O}$ clouds, which float at around $45-70 \mathrm{~km}$ altitudes (Esposito et al., 1983). $\mathrm{H}_{2} \mathrm{SO}_{4}$ is thought to be produced photochemically near the cloud top via the oxidation of $\mathrm{SO}_{2}$, which is abundant below the cloud top, and thus the clouds basically have the characteristics of photochemical aerosols. On the other hand, a strong coupling between cloud condensation and atmospheric motion is expected to occur in the lower part of the cloud layer, where the heating of clouds by upwelling infrared radiation drives vertical convection (Baker and Schubert, 1992; Imamura and Hashimoto, 2001).

The cloud layer absorbs solar radiation, thereby driving various atmospheric motions that might play a central role in the momentum balance of the super-rotation. The super-rotation is a planet-wide easterly wind on Venus; the wind speed increases with height at all latitudes and reaches about $100 \mathrm{~m} \mathrm{~s}^{-1}$ near the cloud top (Schubert et al., 1980). Various mechanisms explaining the super-rotation have been proposed to date (Gierasch et al., 1997). Among them is the combination of a thermally-driven Hadley circulation and large-scale eddies which transports angular momentum equatorward, causing a net upward transport of an-

Copyright (C) The Society of Geomagnetism and Earth, Planetary and Space Sciences (SGEPSS); The Seismological Society of Japan; The Volcanological Society of Japan; The Geodetic Society of Japan; The Japanese Society for Planetary Sciences; TERRAPUB.

doi:10.5047/eps.2011.06.019 gular momentum (Giearsch, 1975; Rossow and Williams, 1979; Iga and Matsuda, 2005). Another candidate is acceleration by thermal tides, which are excited in the cloud layer by periodic solar heating and which propagate vertically to induce momentum exchange between atmospheric layers (Fels and Lindzen, 1974; Newman and Leovy, 1992; Takagi and Matsuda, 2005, 2007). Diurnal and semidiurnal tides are observed in the temperature structure above clouds (Taylor et al., 1980; Zasova et al., 2002; Tellmann et al., 2009) and in the cloud-top wind field (Rossow et al., 1990). The dissipation, at cloud heights, of Kelvin waves that originate in the lower atmosphere might also accelerate the atmosphere (Del Genio and Rossow, 1990; Yamamoto and Tanaka, 1997). Characterization of the dynamics at cloud heights is crucial for understanding the super-rotation.

The cloud-top wind field has been observed by tracking the movements of small-scale ultraviolet features seen in the dayside cloud images taken from Venus orbiters (e.g., Rossow et al., 1990; Moissl et al., 2009). These observations revealed a mean poleward flow with a velocity of up to $10 \mathrm{~m} \mathrm{~s}^{-1}$ at the cloud top. However, the limitation of the local time coverage may allow a significant contamination of the thermal tide component in the estimated meridional circulation, which can be as large as $10 \mathrm{~m} \mathrm{~s}^{-1}$ (Newman and Leovy, 1992). Characterization of the meridional circulation requires wind measurements at all local times.

Eddy motions at the cloud top have also been studied, based mostly on the observations of ultraviolet contrasts, which show various morphological features whose origins are mostly unknown. Among these are the cell-like struc- 


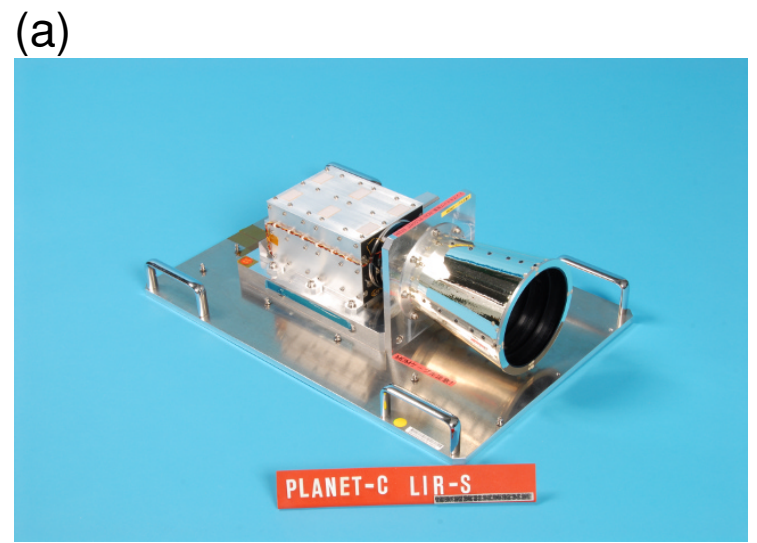

(b)

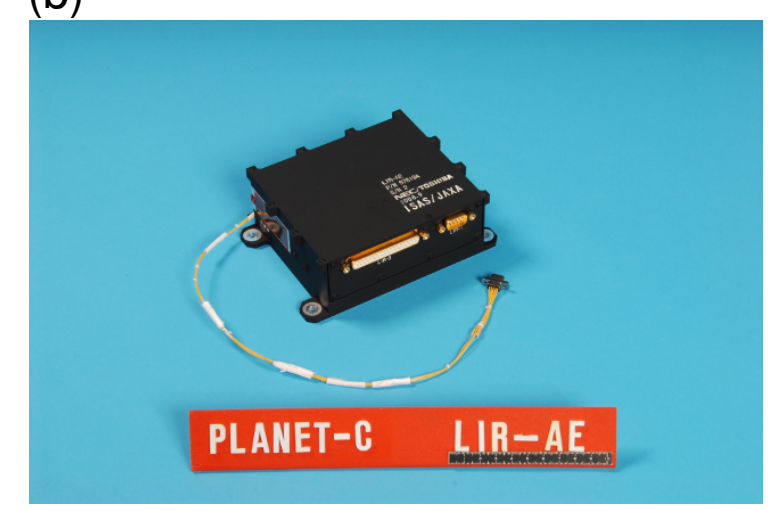

Fig. 1. The flight model of LIR before being assembled to the spacecraft. The sensor unit (a), and the power supply unit (b).

tures seen in the sub-solar region (Rossow et al., 1980; Markiewicz et al., 2007); their horizontal scales of up to several hundreds of kilometers are too large for convective cells, and the cloud-top region is considered to be stably stratified (Baker and Schubert, 1992; Toigo et al., 1994). This mysterious feature might be related to the vertical transport of cloud materials and might ultimately determine the cloud structure.

It should be noted that these ultraviolet features do not necessarily represent the cloud structure, but reflect the horizontal inhomogeneities of ultraviolet absorbers. The solar ultraviolet radiation scattered by the cloud top shows absorption by $\mathrm{SO}_{2}$ at wavelengths shorter than $320 \mathrm{~nm}$ and by unknown materials at longer wavelengths (Esposito et al., 1997). The mixing ratios of both $\mathrm{SO}_{2}$ and the unknown absorber are considered to increase precipitously with decreasing altitude below the cloud top (Pollack et al., 1980; Bertaux et al., 1996), and thus the spatial distributions of these species should also be sensitive to vertical air motions. Such vertical winds may also influence the cloud-top height, but the relation of the cloud height to the vertical wind is uncertain. Recently, cloud altimetry using scattered near-infrared solar radiation showed that ultraviolet dark features tend to correspond to higher clouds in the southern high latitude (Ignatiev et al., 2009). Methods to map the cloud-top height over broad local times and latitudinal regions would further constrain the cloud dynamics.

Efforts to map the thermal emission from the cloud top of Venus have been made using ground-based telescopes
Table 1. Specification of LIR.

\begin{tabular}{ll}
\hline Wavelength region & $8-12 \mu \mathrm{m}$ \\
Field of view & $16.4^{\circ} \times 12.4^{\circ}$ \\
Spacial resolution & $0.05^{\circ}$ \\
Target temperature & $220-250 \mathrm{~K}$ \\
Pixel size & $37 \mu \mathrm{m}$ \\
Number of pixels & $328 \times 248$ \\
NETD & $0.3 \mathrm{~K}($ at $230 \mathrm{~K})$ \\
Absolute accuracy & $3 \mathrm{~K}$ \\
F-number & 1.4 \\
Size & $200 \times 130 \times 110 \mathrm{~mm}$ \\
Weight & $3.5 \mathrm{~kg}$ \\
Power consumption & $29 \mathrm{~W}$ \\
\hline
\end{tabular}

(e.g., Apt et al., 1980) and infrared radiometers onboard the Pioneer Venus orbiter (Taylor et al., 1980) and Venera 15 and 16 (Zasova et al., 2007). They revealed the structures of thermal tides and other planetary-scale waves as well as the polar dipole structure. The visible and Infrared Thermal Imaging Spectrometer (VIRTIS) onboard Venus Express revealed that the temperature distribution in the south polar region is similar to that in the north polar region (Piccioni et al., 2007). However, the observations have been limited in spatial resolution, temporal resolution and the latitudinal coverage, preventing the studies of mesoscale processes and the derivation of wind field by cloud tracking.

Akatsuki, which is the first Japanese Venus orbiter, 


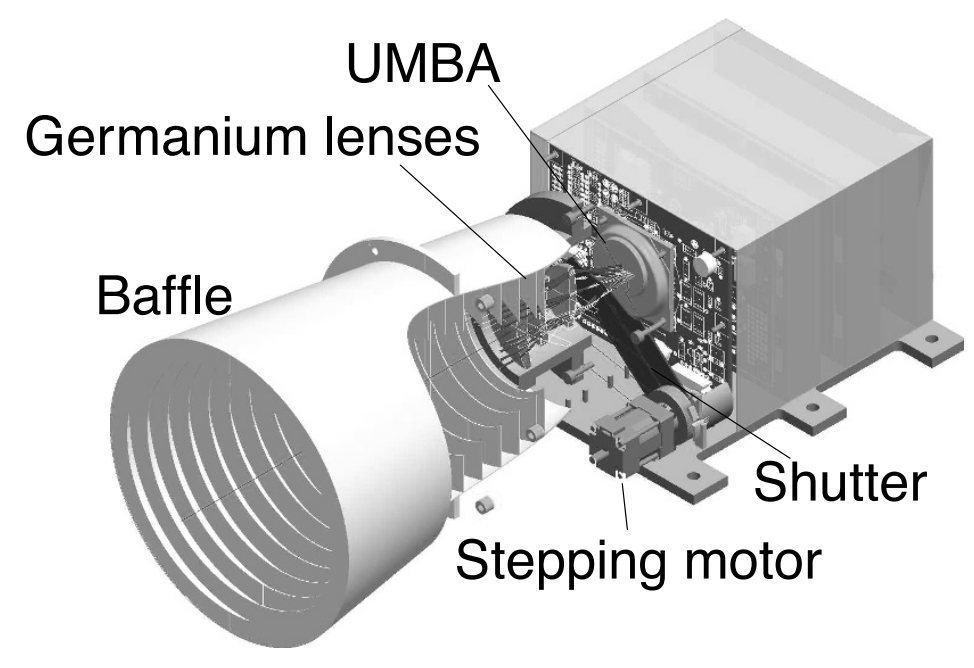

Fig. 2. Schematic of internal structure of the sensor unit of LIR.

aims at understanding the atmospheric dynamics and cloud physics of Venus. Akatsuki maps clouds and minor constituents successively with four cameras covering wavelengths from infrared to ultraviolet, detects lightning flashes with a high-speed photometer, and retrieves the vertical structure of the atmosphere with a radio occultation technique (Nakamura et al., 2007, 2011). The planned orbit around Venus is a 30-hour-period elliptical orbit (370$78,500 \mathrm{~km}$ ) near the ecliptic plane, and cloud images will be obtained every 2 hours for each observation wavelength. Science instruments altogether observe multiple height levels of the atmosphere to model the three-dimensional structure and dynamics. Among them is the Longwave Infrared Camera (LIR), which maps the thermal emission from the cloud top at 8-12 $\mu \mathrm{m}$ wavelengths (Taguchi et al., 2007). Unlike other cameras onboard Akatsuki, LIR is able to take images of dayside and nightside clouds with equal quality. This is advantageous not only to the studies of the diurnal cycles of cloud processes but also to the precise determination of the zonal-mean meridional circulation.

\section{Instrument Design}

The global-averaged temperature field obtained from the Venera 15 IR spectrometry data (Zasova et al., 2007) suggests that the temperature range of the structure is between 205 and $260 \mathrm{~K}$, and the numerical study of penetrative convection about the Venusian cloud between 40 and $60 \mathrm{~km}$ altitude has derived potential temperature profiles which suggest the range of cloud-top altitude is within several kilometers (Baker et al., 1998; Ignatiev et al., 2009). In order to detect a cloud-height difference of a few hundred meters, LIR requires $0.3 \mathrm{~K}$ of noise equivalent temperature difference (NETD), which can be regarded as the temperature resolution, for a $230 \mathrm{~K}$ target as shown in Table 1 . An uncooled micro-bolometer array (UMBA) (Tanaka et al., 2000) for a commercial infrared camera is used for LIR as an image sensor. The commercial camera is designed to give the best performance when it views roomtemperature objects (Wada et al., 1998). For LIR, the electronics and the driving parameters must be optimized for the low-temperature targets of this mission. The UMBA has $328 \times 248$ pixels with a pixel size of $37 \mu \mathrm{m}$, and its temperature is stabilized at $313 \mathrm{~K}$ by a Peltier temperature control system. Since an UMBA does not need cryogenic apparatus, which is commonly used for photodiode-type infrared detectors onboard spacecraft, LIR is relatively small and light in weight; it achieves a size of $200 \times 130 \times 110$ $\mathrm{mm}$, a weight of $3.5 \mathrm{~kg}$ and a power consumption of 29 W. UMBAs have been applied to a couple of space missions: the ISIR instrument onboard Space Shuttle (STS-85) (Lancaster et al., 2001) and the THEMIS instrument onboard Mars Odyssey (Christensen et al., 2004). LIR will be a benchmark of an infrared camera with an UMBA for future space explorations to Mars, asteroids and the Earth.

The internal structure and block diagram of LIR are shown in Figs. 2 and 3, respectively. LIR consists of a sensor unit LIR-S which manages the function of image acquisition, an external power supply unit LIR-AE which converts the primary electric power to several voltages to distribute them to a regulator and a mechanical shutter, and a baffle which keeps direct sunlight away from the optical aperture. The optics in the sensor unit consists of three germanium lenses with the F-number of 1.4. The field-of-view of $16.4^{\circ} \times 12.4^{\circ}$ is based on a common requirement with other cameras. The pixel resolution of $0.05^{\circ}$ corresponds to $26-70 \mathrm{~km}$ on the Venus surface when the spacecraft views Venus from distances of $3-8 \times 10^{4} \mathrm{~km}$ on an elliptical orbit. The temperature of the optics is kept within 293-308 K by the heater-controlling electronics (HCE) of the spacecraft in order to prevent thermal distortions. The mechanical shutter which works not only as a sunlight shield but also as a blackbody for calibration is positioned just in front of the UMBA and is driven by a stepping motor. A bandpass filter whose profile is shown in Fig. 4 is inserted at the pupil position of the optics. The UMBA for LIR has large pixelto-pixel inhomogeneities of offset and sensitivity. Although these inhomogeneities are partly reduced in the analog circuit before analog-to-digital conversion, by using the onchip fixed pattern noise (OFPN) data which is preacquired before launch, they still remain in the raw data. In order 


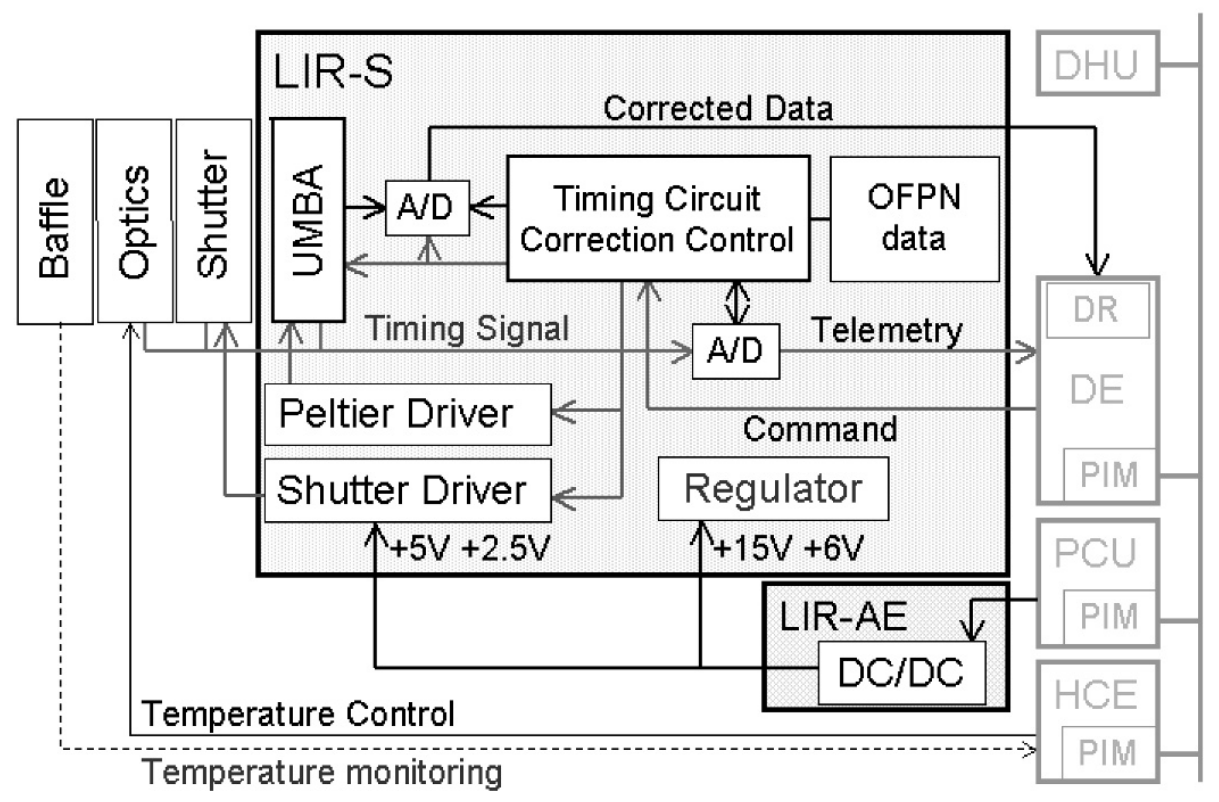

Fig. 3. The block diagram of LIR when configurated in the space craft.

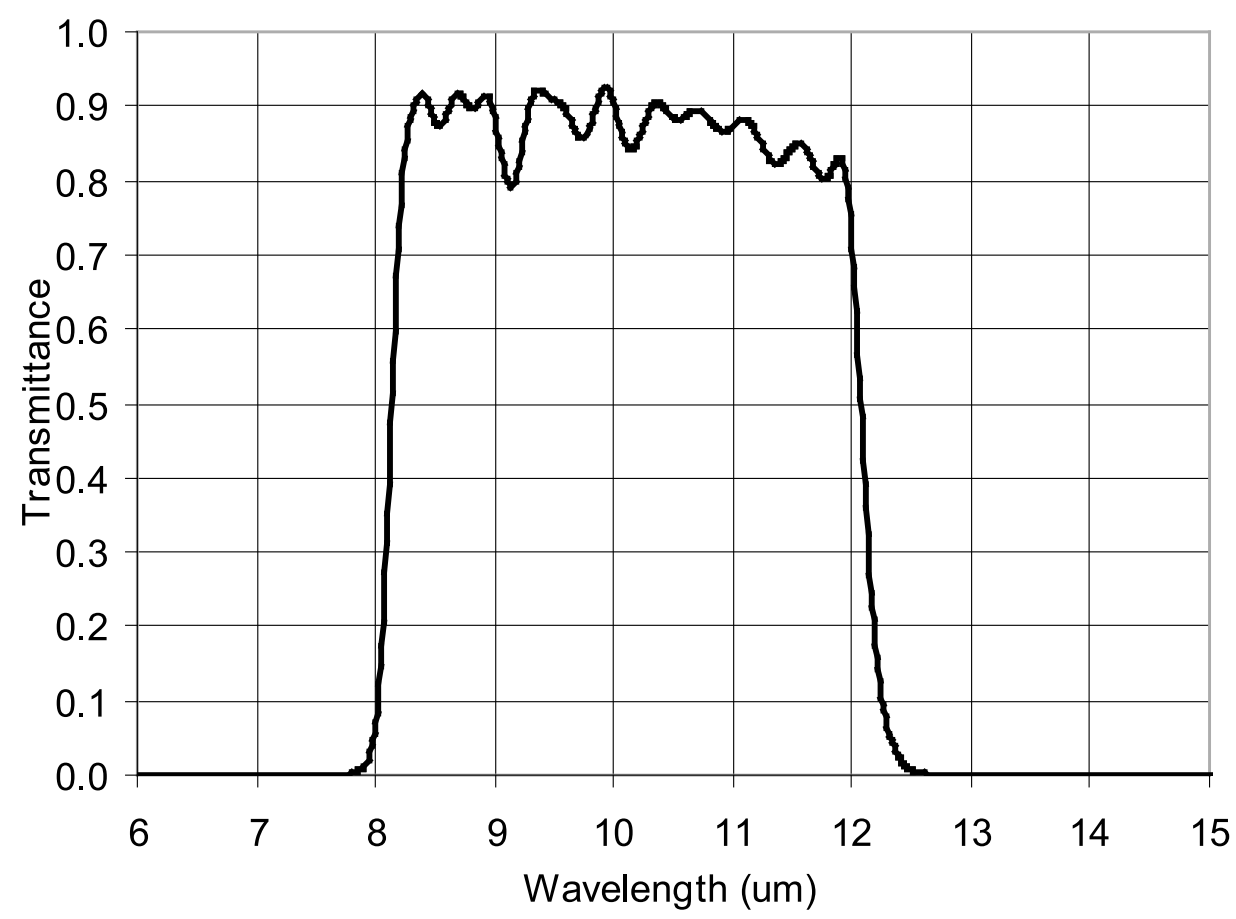

Fig. 4. The transmittance spectrum for the bandpass filter incorporated in LIR. The angle of incidence is zero.

to remove the pixel-dependent offset, LIR acquires a target data and a shutter data sequentially, and the shutter data is subtracted from the target data as shown in Fig. 5. All target and shutter images that are used in this subtraction are generated by averaging up to 128 raw images (first averaging), that are acquired continuously at a frame rate of $60 \mathrm{~Hz}$. Then up to 32 offset-removed images are acquired within several minutes and averaged (second averaging). The stability of the spacecraft's attitude, which is designed for the other cameras onboard Akatsuki, is within $0.015 \mathrm{deg}$
(Nakamura et al., 2011). Since they have a higher spatial resolution than LIR, there is no restriction for the observation of LIR.

The control of LIR for sequential image acquisition and the subsequent data processing such as averaging and offset subtraction are performed by the Digital Electronics (DE), which is an onboard controller for four cameras including LIR (Nakamura et al., 2007). The amount of data transfer to the ground station is reduced by DE using a lossless data compression algorithm called HIREW (Takada et al., 


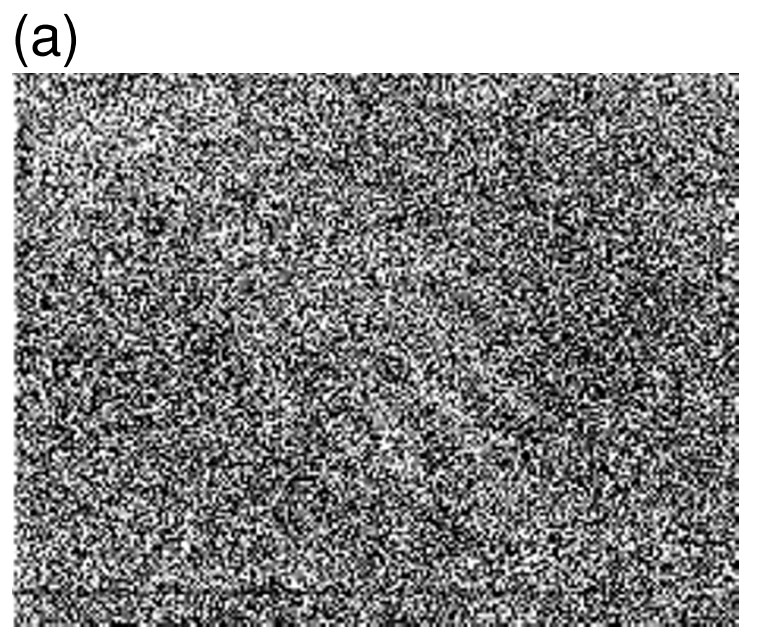

\section{(b)}

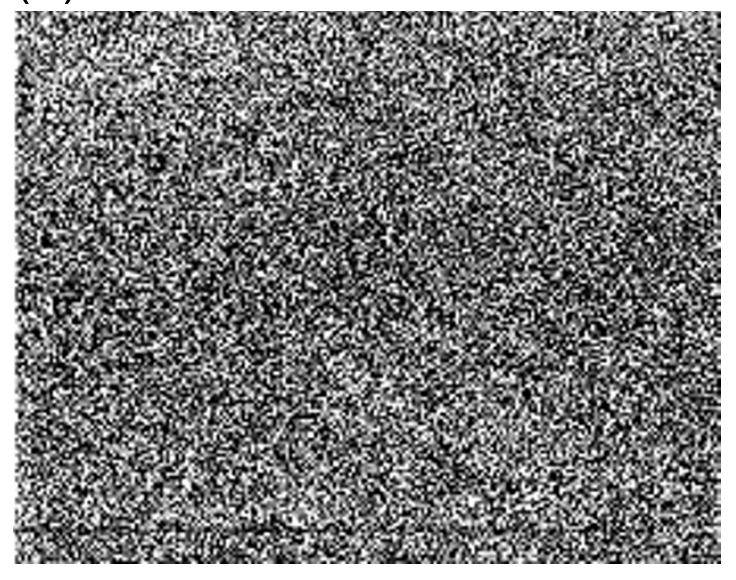

(c)

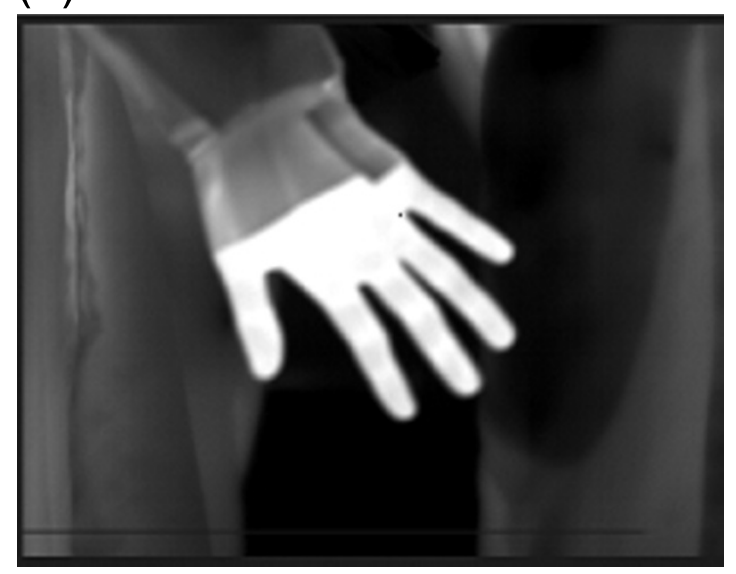

Fig. 5. Example of infrared images acquired by the bread-board model of LIR. A raw target image (a), a raw shutter image for calibration (b), and the resultant image produced by subtracting the shutter image from the target image (c).

2007). There still remains a slight inhomogeneity of the sensitivity, even in a subtracted image. This will be corrected after being transmitted to the ground. The resultant image, which gives the difference of the brightness temperature between the object and the shutter, is further converted to a brightness temperature map by adding the temperature of the shutter to all pixel values.

\section{Evaluation Test of the Flight Model}

3.1 Measurement of the NETD

The NETD of LIR has been evaluated in a vacuum chamber in which the pressure was maintained in the range between $10^{-6}$ and $10^{-8}$ torr. The configuration of equipment in the chamber is shown in Fig. 6. An aluminum table, whose temperature is controlled by liquid nitrogen and an electric heater, is placed at the bottom in the chamber. 


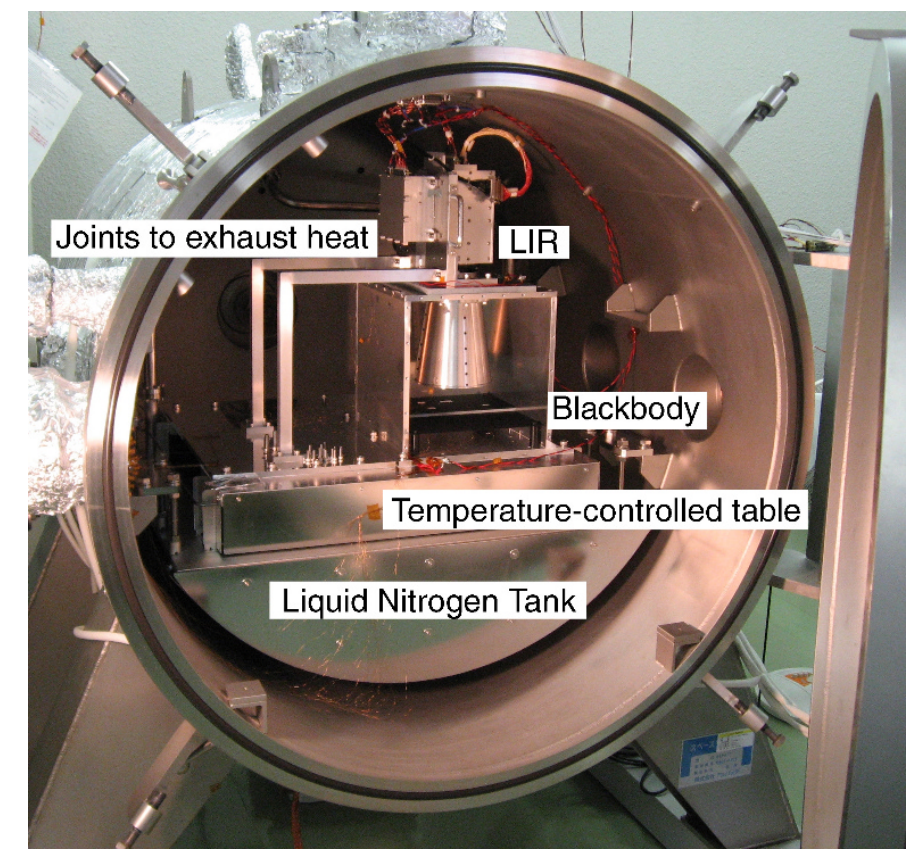

Fig. 6. Vacuum chamber in which equipments are configured for image acquisition by LIR.

An aluminum plate fixed on the temperature-controlled table works as a blackbody with an emissivity of $0.85-0.92$ at room temperature. The blackbody is radiatively coupled with the temperature-controlled table and shrouded by aluminum plates for stabilizing the temperature within $230 \pm 0.1 \mathrm{~K}$. LIR was placed above the aluminum plate shroud, and oriented downward. The operational heat of LIR is removed to the table via aluminum joints and kept at $300 \mathrm{~K}$, which is a nominal temperature in the orbit.

Two kinds of blackbodies were used for the evaluation: one is a combination of two panels which are arranged side by side and maintained at slightly different temperatures using a film heater, and the other is a plate with a homogeneous temperature. Figure 7(a) and (b) show images of these blackbodies without the secondary image accumulation. Brightening of the edges of the images is obvious both in (a) and (b). Usually in visible light photographs, vignetting of optics causes darkening of the edges of a photo. However, when a low-temperature object is imaged by infrared, the radiation emitted by the object and reaching the detector is overwhelmed by the radiation from the optics and its mount whose temperature is higher than that of the object. Therefore, vignetting results in a brightening of the image of the cold object. The temperature difference of $4.7 \mathrm{~K}$ between the warm (right) and cold (left) side is not apparent in (a). The vignetting effect was corrected by subtracting the image of the uniform blackbody (b). The result (Fig. 7(c)) shows that the temperature contrast was successfully detected although the boundary was blurred because of defocus. When observing Venus, the brightening of the edges will be removed by the subtraction of uniform blackbody images acquired in the vacuum chamber, or deep-space images that can be acquired during the orbit of Akatsuki.

NETD will be improved by averaging several tens of im- ages taken within a few minutes (Geoffray et al., 2000). Figure 8 shows the NETD variations with the numbers of the first and secondary accumulations. They are estimated as NETD $=d T /(S / N)$, where $d T$ is the actual temperature difference between the warmer and colder blackbodies, $S$ the difference of the observed mean brightness between the warmer and colder blackbodies in the image, and $N=\sigma$ the standard deviation of the observed brightness that is evaluated locally in the warmer or colder domains. The reduction of noise by the first accumulation is effective till $m=32$ as long as the number of the second accumulation $n$ is less than 5. When the number of second averaging is five or more, the noise compression becomes negligible. This suggests that the noise is not ideally random. When the first and second averaging were 32 and 32, respectively, substituting the observed values of $d T=4.22 \mathrm{~K}, S=2922$ and $N=247$ into the equation above, we obtained the best NETD $\sim 0.36 \mathrm{~K}$, which meets the required specification. These numbers of averaging have been adopted for the flight model.

\subsection{Endurance test of the shutter}

The stepping motor used for driving the shutter requires to be of high reliability. Since the number of operations in a mission period is expected to be a maximum of 120,000 times, a trial endurance test has been carried out in a vacuum environment using a shutter component. The motor has attained an operation of 240,000 times for about one month with round-the-clock operation.

\subsection{Tolerance of the UMBA to vibration, radiation and sunlight}

The tolerance of the UMBA to the launch environment was evaluated by using a vibration testing machine. Random vibration levels that were calculated using a mathematical structure model of LIR were imposed on the UMBA for 45 seconds for three axes; the spectrally-integrated lev- 


\section{(a)}

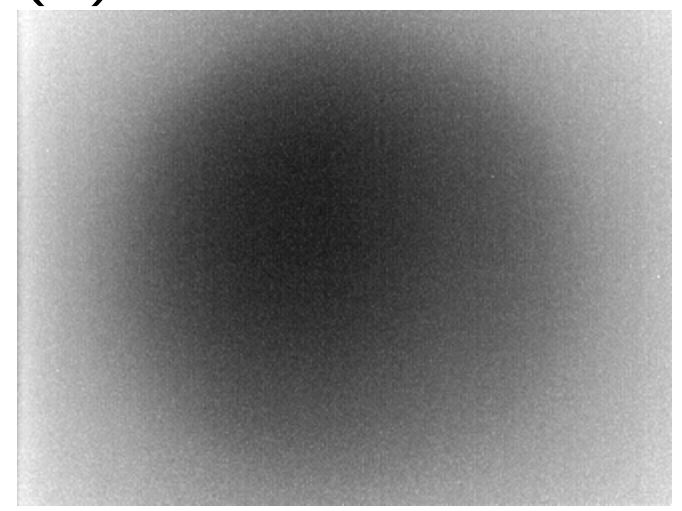

\section{(b)}

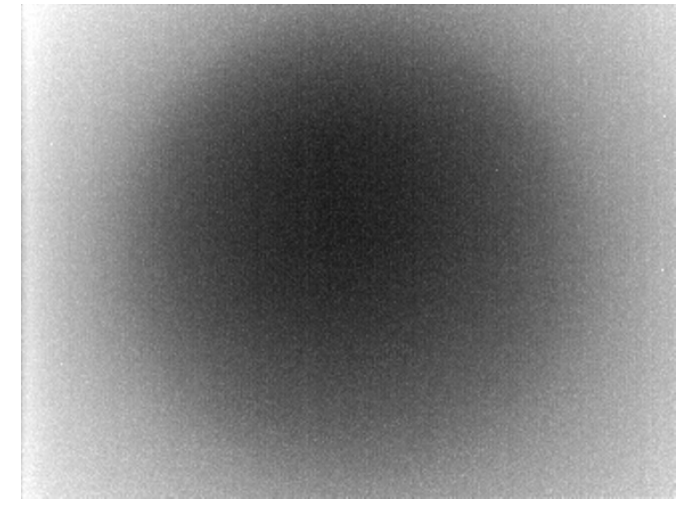

(c)

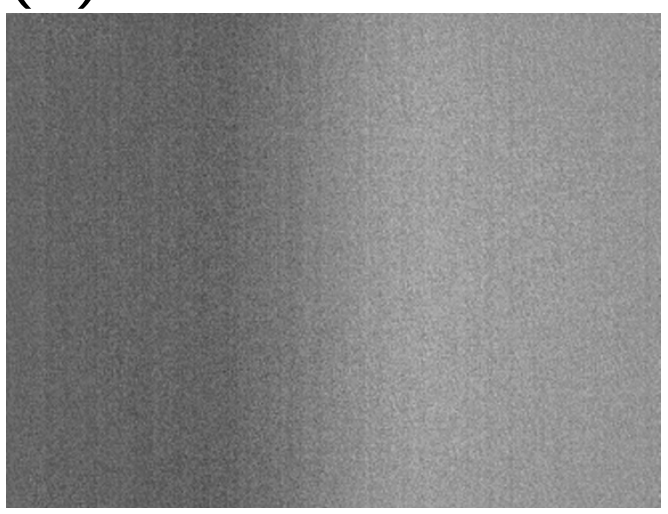

Fig. 7. Images of blackbodies acquired by LIR in a vacuum environment. (a) Temperature of $\sim 230 \mathrm{~K}$ with the right half being $4.7 \mathrm{~K}$ warmer than the left, (b) Temperature of $\sim 230 \mathrm{~K}$ without temperature contrast, (c) Same as (a), but the brightness distribution for a uniform target (b) has been subtracted.

els were several tens of Grms. The function of the UMBA was normal after the vibration test without any pixel defects or malfunctions.

Since this is the first time for this type of UMBA to be used in the space environment, tolerance of the UMBA to high-energy protons was evaluated by exposing the UMBA to a $100 \mathrm{MeV}$ proton beam at the rate of $4 \mathrm{~Gy} / \mathrm{min}$ with a total dose of $300 \mathrm{~Gy}$ which exceeds what is expected during the mission life. The UMBA was set active during the test and no malfunction was observed during the exposure to the proton beam. After the exposure, the UMBA was inspected and shown to have the nominal sensitivity, NETD and no pixel defects.

At the time of launch the shutter of LIR is closed. Vibration and shock tests show that the shutter remained closed even after the mechanical perturbations expected for the launch with margins applied. Moreover, a shutter-closing command will be issued as soon as possible after separation of the spacecraft from the launch vehicle in the case of an accidental opening of the shutter. However, there is a slight possibility that vibration or shock will cause the shutter to open at the time of launch and direct sunlight may enter the 


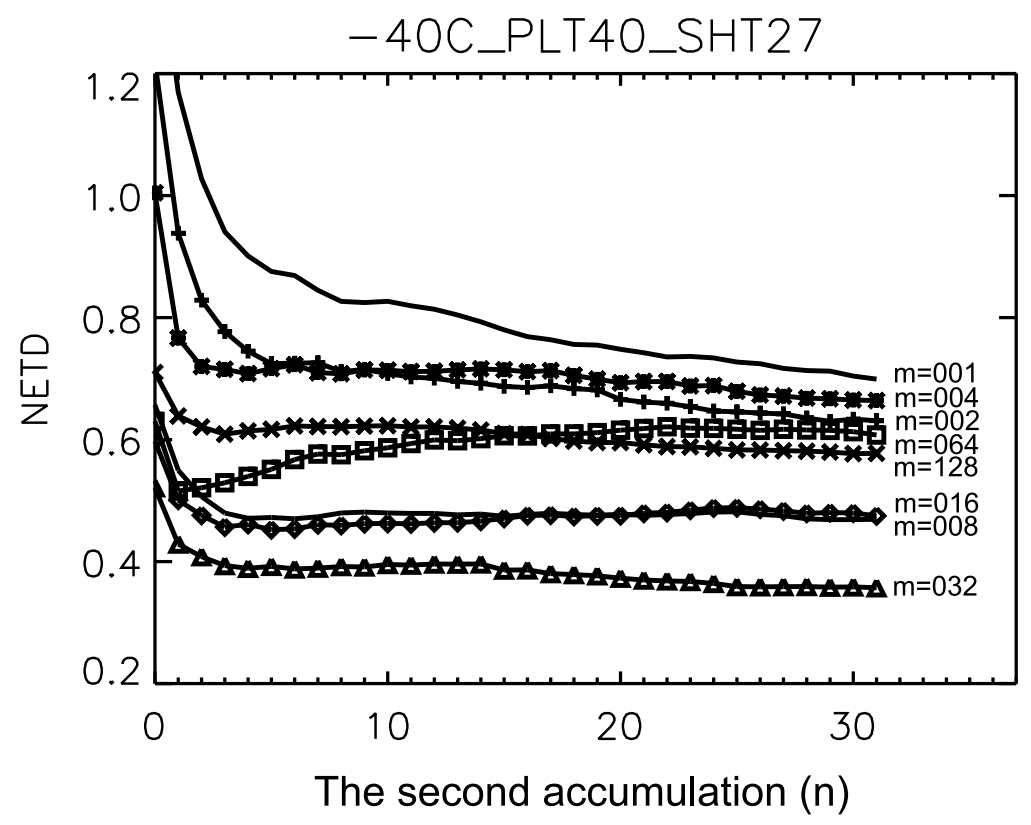

Fig. 8. Variations of the NETD accompanying change of number of the first $(m)$ and second $(n)$ accumulation. They were estimated from images in which the temperature of the blackbodies was $230 \mathrm{~K}$.

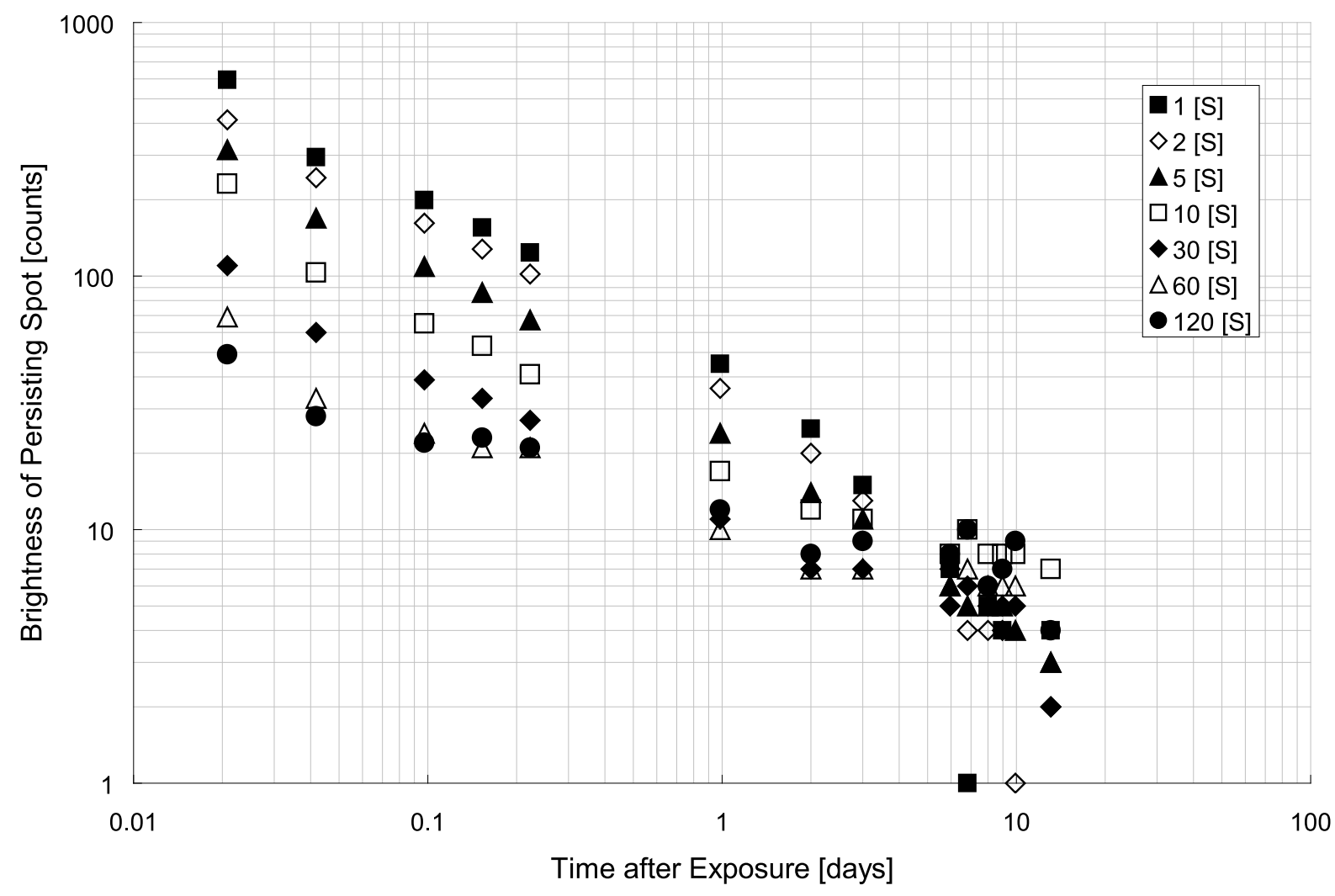

Fig. 9. Time variations of luminosity of afterimages after direct sunlight inputs. The time of the sunlight input was varied in steps between 1 second (minimum) to 2 minutes (maximum).

aperture of LIR for less than two minutes until attitude and orbit control is started. In such a case assumed, the tolerance of the UMBA to direct sunlight input was evaluated by using a trial piece beforehand because $6000 \mathrm{~K}$ blackbody radiation at the wavelength of $10 \mu \mathrm{m}\left(4348 \mathrm{~W} \mathrm{~m}^{-2}\right.$ $\operatorname{str}^{-1} \mu \mathrm{m}^{-1}$ ) is much greater than the $300 \mathrm{~K}$ radiation (10 $\mathrm{W} \mathrm{m}{ }^{-2} \operatorname{str}^{-1} \mu \mathrm{m}^{-1}$ ). Figure 9 shows the time variations in brightness of afterimages of the sun after sunlight input of which the maximum duration is two minutes. Although the brightness becomes larger as the time duration of sunlight 
input becomes longer, they decrease to around seven counts that are negligible values after ten days. The UMBA does not seem to suffer obvious or permanent damage by the sunlight input of at least two minutes. It is supposed that the afterimages of strong light input are caused by the changes of the physical properties of the bolometer due to the strong heat inputs, and that they will disappear as these physical properties recover with time.

\subsection{Temperature control of camera}

As stated in the previous section, the UMBA detects not only the thermal radiation from the Venusian atmosphere, but also that from the lens and optical mount. The temperatures of lens and optical mount must be controlled so that their influences on an image are minimized. It has been estimated by calculation, using the nominal transmittance of the lens, that a stability of $\pm 2.3 \mathrm{~K}$ is required for the lens temperature, which is almost equivalent to the optical mount temperatures. In order to confirm the temperature range requirement, the flight model of LIR took images of a uniform temperature blackbody under different thermal conditions. Both of the signal outputs for the shutter and the object must be covered simultaneously by the 12 bits dynamic range of the $\mathrm{A} / \mathrm{D}$ converter. It is concluded that if the optical mount temperature is within 293.5-304.6 K, LIR can take an image of an object whose temperature is as low as $203 \mathrm{~K}$.

\section{Summary}

LIR developed for Akatsuki, the first Japanese Venus Climate Orbiter, the first lightweight infrared camera to use an uncooled micro-bolometer array as an image sensor. The infrared camera observes the thermal emission from the cloud top of Venus in the wavelength region $8-12 \mu \mathrm{m}$ to obtain two-dimensional distributions of temperature and wind vector at the cloud top, where the temperature is typically as low as $230 \mathrm{~K}$. The flight model of LIR has been manufactured and its performance was confirmed by several tests in a vacuum environment. It has been shown that the target performance of NETD $\sim 0.3 \mathrm{~K}$ at an object temperature of $\sim 230 \mathrm{~K}$ is achieved by averaging several tens of images acquired within a few minutes. This NETD corresponds to a height difference at a cloud top of $100 \mathrm{~m}$ for an average temperature profile. The camera-case temperature should be stabilized within the temperature range of 293.5$304.6 \mathrm{~K}$ in order to take an image of an object having a temperature as low as $203 \mathrm{~K}$. The tolerances of the bolometer array to sunlight, the mechanical environment, and highenergy protons, have been tested and satisfy the requirements. It is also confirmed that the shutter mechanism has enough endurance for the mission life of two years.

LIR has been mounted on Akatsuki which was launched on 21 May, 2010. LIR and the other cameras onboard Akatsuki have taken images of the Earth immediately after the launch. Furthermore, LIR has taken images of deep space for calibrations of the UMBA. However, the Venus orbit insertion maneuver for Akatsuki on December 7, 2010, failed. At present the spacecraft is orbiting the Sun, and it will have a chance to encounter Venus in 5 or 6 years time. JAXA is examining the possibility of conducting an orbit insertion maneuver again at this opportunity.
Acknowledgments. The authors wish to thank Mr. Higashino, Mr. Kashikawa and other engineers of NT Space Co. Inc. for their efforts in manufacturing LIR.

\section{References}

Apt, J., R. A. Brown, and R. M. Goody, The character of the thermal emission from Venus, J. Geophys. Res., 85, 7934-7940, 1980.

Baker, R. D. and G. Schubert, Cellular convection in the atmosphere of Venus, Nature, 355, 710-712, 1992.

Baker, R. D., G. Schubert, and P. W. Jones, Cloud-level penetrative compressive convection in the Venus atmosphere, J. Atmos. Sci., 55(1), 317, 1998.

Bertaux, J., T. Widemann, A. Hauchecorne, V. I. Moroz, and A. P. Ekonomov, VEGA 1 and VEGA 2 entry probes: An investigation of local UV absorption (220-400 nm) in the atmosphere of Venus (SO2, aerosols, cloud structure), J. Geophys. Res., 101(E5), 12709-12746, 1996.

Christensen, P. R., B. M. Jakosky, H. H. Kieffer, M. C. Malin, H. Y. McSween, K. Nealson, G. L. Mehall, S. H. Silverman, S. Ferry, M. Caplinger, and M. Ravine, The Thermal Emission Imaging System (THEMIS) for the Mars 2001 Odyssey Mission, Space Sci. Rev., 110, 85-130, 2004.

Del Genio, A. D. and W. B. Rossow, Planetary-scale waves and the cyclic nature of cloud top dynamics on Venus, J. Atmos. Sci., 47, 293-318, 1990.

Esposito, L. W., R. G. Knollenberg, M. Y. Marov, O. B. Toon, and R. P. Turco, The clouds and hazes of Venus, in Venus, edited by Hunten, D. M., L. Colin, T. M. Donahue, and V. I. Moroz, University of Arizona Press, 1983.

Esposito, L. W., J. L. Bertaux, V. Krasnopolsky, V. I. Moroz, and L. V. Zasova, Chemistry of lower atmosphere and clouds, in Venus II, edited by Bougher, S., D. Hunten, and R. Phillips, University of Arizona Press, 1997.

Fels, S. and R. S. Lindzen, Interaction of thermally excited gravity waves with mean flows, Geophys. Fluid Dyn., 6, 149-191, 1974.

Geoffray, H., A. Bardoux, M. Laporte, and J. Tissot, Uncooled infrared microbolometer arrays for Earth remote sensing, Proc. SPIE, 4130, 527-536, 2000.

Gierasch, P. J., Meridional circulation and the maintenance of the Venus atmospheric rotation, J. Atmos. Sci., 32, 1,038-1,044, 1975.

Gierasch, P. J. et al., The general circulation of the Venus atmosphere: An assessment, in Venus II, edited by Bougher, S., D. Hunten, and R. Phillips, 459-500, University of Arizona Press, Tucson, 1997.

Iga, S. and Y. Matsuda, Shear instability in a shallow water model with implication for the Venus atmosphere, J. Atmos. Sci., 62, 2514-2527, 2005.

Ignatiev, N. I., D. V. Titov, G. Piccioni, P. Drossart, W. J. Markiewicz, V. Cottini, Th. Roatsch, M. Almeida, and N. Manoel, Altimetry of the Venus cloud tops from the Venus Express observations, J. Geophys. Res., 114, E00B43, doi:10.1029/2008JE003320, 2009.

Imamura, T. and G. L. Hashimoto, Microphysics of Venusian clouds in rising tropical air, J. Atmos. Sci., 58, 3597-3612, 2001.

Lancaster, R. S., K. Manizade, S. P. Palm, J. D. Spinhirne, and V. S. Scott, The Compact Visible and Infrared Radiometer (COVIR) for Earth and climate monitoring, Proc. IEEE, 4, 1719-1727, 2001.

Markiewicz, W. J., D. V. Titov, S. S. Limaye, H. U. Keller, N. Ignatiev, R. Jaumann, N. Thomas, H. Michalik, R. Moissl, and P. Russo, Morphology and dynamics of the upper cloud layer of Venus, Nature, 450, doi:10.1038/nature06320, 2007.

Moissl, R., I. Khatuntsev, S. S. Limaye, D. V. Titov, W. J. Markiewicz, N. I. Ignatiev, T. Roatsch, K.-D. Matz, R. Jaumann, M. Almeida, G. Portyankina, T. Behnke, and S. F. Hviid, Venus cloud top winds from tracking UV features in Venus Monitoring Camera images, J. Geophys. Res., 114, E00B31, doi:10.1029/2008JE003117, 2009.

Nakamura, M., T. Imamura, M. Ueno, N. Iwagami, T. Satoh, S. Watanabe, M. Taguchi, Y. Takahashi, M. Suzuki, T. abe, G. L. Hashimoto, T. Sakanoi, S. Okano, Y. Kasaba, J. Yoshida, M. Yamada, N. Ishii, T. Yamada, K. Uemizu, T. Fukuhara, and K.-I. Oyama, Planet-C: Venus Climate Orbiter mission of Japan, Planet. Space Sci., 55, 1831-1842, 2007.

Nakamura, M., T. Imamura, N. Ishii, T. Abe, T. Satoh, M. Suzuki, M. Ueno, A. Yamazaki, N. Iwagami, S. Watanabe, M. Taguchi, T. Fukuhara, Y. Takahashi, M. Yamada, N. Hoshino, S. Ohtsuki, K. Uemizu, G. L. Hashimoto, M. Takagi, Y. Matsuda, K. Ogohara, N. Sato, Y. Kasaba, T. Kouyama, N. Hirata, R. Nakamura, Y. Yamamoto, N. Okada, 
T. Horinouchi, M. Yamamoto, and Y. Hayashi, Overview of Venus orbiter, Akatsuki, Earth Planets Space, 63, 443-457, 2011.

Newman, M. and C. Leovy, Maintenance of strong rotational winds in Venus' middle atmosphere by thermal tides, Science, 257, 647-650, 1992.

Piccioni, G., P. Drossart, A. Sanchez-Lavega, R. Hueso, F. Taylor, C. Wilson, D. Grassi, L. Zasova, M. Moriconi, A. Adriani, S. Lebonnois, A. Coradini, B. Bezard, F. Angrilli, G. Arnold, K. H. Baines, G. Bellucci, J. Benkhoff, J. P. Bibring, A. Blanco, M. I. Blecka, R. W. Carlson, A. Di Lellis, T. Encrenaz, S. Erard, S. Fonti, V. Formisano, T. Fouchet, R. Garcia, R. J. Haus, J. Helbert, N. I. Ignatiev, P. Irwin, Y. Langevin, M. A. Lopez-Valverde, D. Luz, L. Marinangeli, V. Orofino, A. V. Rodin, M. C. Roos-Serote, B. Saggin, D. M. Stam, D. Titov, G. Visconti, and M. Zambelli, South-polar features on Venus similar to those near the north pole, Nature, 450, doi:10.1038/nature06209, 2007.

Pollack, J. B., O. B. Toon, R. C. Whitten, R. Boese, B. Ragent, M. Tomasko, L. Esposito, L. Travis, and D. Wiedman, Distribution and source of the UV absorption in Venus' atmosphere, J. Geophys. Res., 85, 8141-8150, 1980.

Rossow, W. B. and G. P. Williams, Large-scale motion in the Venus stratosphere, J. Atmos. Sci., 36, 377-389, 1979.

Rossow, W. B., A. Del Genio, S. Limaye, L. Travis, and P. Stone, Cloud morphology and motions from Pioneer Venus images, J. Geophys. Res., 85, 8107-8128, 1980.

Rossow, W. B., A. D. Del Genio, and T. Eichler, Cloud-tracked winds from Pioneer Venus OCPP images, J. Geophys. Res., 47, 2053-2084, 1990.

Schubert, G., C. Covey, A. Del Genio, L. S. Elson, G. Keating, A. Seiff, R. E. Young, J. Apt, C. C. Counselman III, A. J. Kliore, S. S. Limaye, H. E. Revercomb, L. A. Sromovsky, V. E. Suomi, F. Taylor, R. Woo, and U. von Zahn, Structure and circulation of the Venus atmosphere, $J$. Geophys. Res., 85, 8007-8025, 1980.

Taguchi, M., T. Fukuhara, T. Imamura, M. Nakamura, N. Iwagami, M. Ueno, M. Suzuki, G. L. Hashimoto, and K. Mitsuyama, Longwave Inflared Camera onboard the Venus Climate Orbiter, Adv. Space Res., 40, 861-868, 2007.

Takada, J., S. Senda, H. Hihara, M. Hamai, T. Oshima, S. Hagino, M. Suzuki, and S. Ichikawa, A fast progressive lossless image compression method for space and satellite images, Geoscience and Remote Sensing Symposium, IGARSS 2007, IEEE International, 479-481, 2007.
Takagi, M. and Y. Matsuda, Sensitivity of thermal tides in the Venus atmosphere to basic zonal flow and Newtonian cooling, Geophys. Res. Lett., 32, doi:10.1029/2004GL022060, 2005.

Takagi, M. and Y. Matsuda, Effects of thermal tides on the Venus atmospheric superrotation, J. Geophys. Res., 112, D09112, doi:10.1029/2006JD007901, 2007.

Tanaka, A., K. Chiba, T. Endoh, K. Okuyama, A. Kawahara, K. Iida, and N. Tsukamoto, Low-noise readout circuit for uncooled infrared FPA, Proc. SPIE, 4130, 160-167, 2000.

Taylor, F. W., R. Beer, M. T. Chahine, D. J. Diner, L. S. Elson, R. D. Haskins, D. J. McCleese, J. V. Martonchik, P. E. Reichley, S. P. Bradley, J. Delderfield, J. T. Schofield, C. B. Farmer, L. Froidevaux, J. Leung, M. T. Coffey, and J. C. Gille, Structure and meteorology of the middle atmosphere of Venus Infrared remote sensing from the Pioneer orbiter, J. Geophys. Res., 85, 7963-8006, 1980.

Tellmann, S., M. Pätzold, B. Häusler, M. K. Bird, and G. L. Tyler, Structure of the Venus neutral atmosphere as observed by the Radio Science experiment VeRa on Venus Express, J. Geophys. Res., 114, E00B36, doi:10.1029/2008JE003204, 2009.

Toigo, A., P. J. Gierasch, and M. D. Smith, High resolution cloud feature tracking on Venus by Galileo, Icarus, 109, 318-336, 1994.

Wada, H., M. Nagashima, N. Oda, T. Sasaki, A. Kawahara, M. Kanzaki, Y. Tsuruta, T. Mori, S. Matsumoto, T. Shima, M. Hijikawa, N. Tsukamoto, and H. Gotoh, Design and performance of 256x256 Bolometer-Type uncooled infrared detector, Proc. SPIE, 3379, 90-99, 1998.

Yamamoto, M. and H. Tanaka, Formation and maintenance of the 4-day circulation in the Venus middle atmosphere, J. Atmos. Sci., 54, 14721489, 1997.

Zasova, L., I. V. Khatountsev, N. I. Ignatiev, and V. I. Moroz, Local time variations of the middle atmosphere of Venus: solar-related structures, Adv. Space Res., 29(2), 243-248, 2002.

Zasova, L. V., N. Ignatiev, I. Khatuntsev, and V. Linkin, Structure of the Venus atmosphere, Planet. Space Sci., 55, 1712-1728, 2007.

T. Fukuhara (e-mail: tetsuyaf@ep.sci.hokudai.ac.jp), M. Taguchi, T. Imamura, M. Nakamura, M. Ueno, M. Suzuki, N. Iwagami, M. Sato, K. Mitsuyama, G. L. Hashimoto, R. Ohshima, T. Kouyama, H. Ando, and M. Futaguchi 